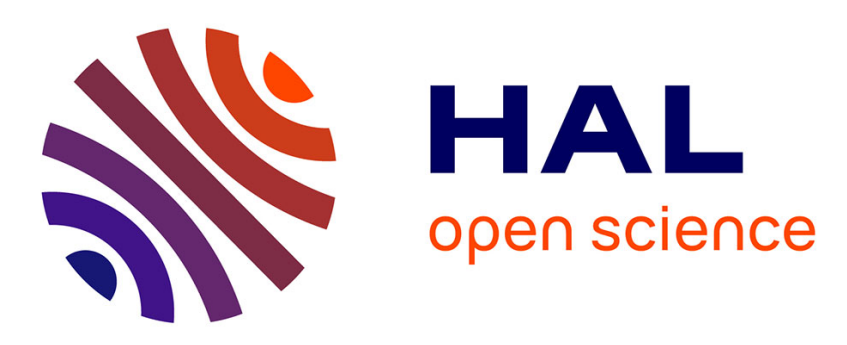

\title{
Dynamics of laser-induced cavitation bubbles at a solid-liquid interface in high viscosity and high capillary number regimes
}

Tim Hupfeld, Gaétan Laurens, Samy Merabia, Stephan Barcikowski, Bilal Gökce, David Amans

\section{To cite this version:}

Tim Hupfeld, Gaétan Laurens, Samy Merabia, Stephan Barcikowski, Bilal Gökce, et al.. Dynamics of laser-induced cavitation bubbles at a solid-liquid interface in high viscosity and high capillary number regimes. Journal of Applied Physics, 2020, 127 (4), pp.044306. 10.1063/1.5116111 . hal-03035284

\author{
HAL Id: hal-03035284 \\ https://hal.science/hal-03035284
}

Submitted on 5 Dec 2020

HAL is a multi-disciplinary open access archive for the deposit and dissemination of scientific research documents, whether they are published or not. The documents may come from teaching and research institutions in France or abroad, or from public or private research centers.
L'archive ouverte pluridisciplinaire HAL, est destinée au dépôt et à la diffusion de documents scientifiques de niveau recherche, publiés ou non, émanant des établissements d'enseignement et de recherche français ou étrangers, des laboratoires publics ou privés. 


\title{
Dynamics of laser-induced cavitation bubbles at a solid-liquid interface in high viscosity and high capillary number regimes
}

\author{
Tim Hupfeld ${ }^{1}$, Gaétan Laurens ${ }^{2}$, Samy Merabia², Stephan Barcikowski ${ }^{1}$, Bilal Gökce ${ }^{1^{*}}$, and David Amans ${ }^{2^{*}}$ \\ ${ }^{1}$ Technical Chemistry $\mathrm{I}$ and Center for Nanointegration Duisburg-Essen (CENIDE), University of Duisburg-Essen, \\ Universitaetsstrasse 7, 45141 Essen, Germany \\ 2 Univ Lyon, Univ Claude Bernard Lyon 1, CNRS, Institut Lumière Matière, F-69622, Villeurbanne, France.
}

*corresponding authors: bilal.goekce@uni-due.de, david.amans@univ-lyon1.fr

\begin{abstract}
No unified model is available yet to explain the dynamics of laser-induced cavitation bubbles during laser ablation of solid targets in liquids, when an extremely high capillary number is achieved $(>100)$, i.e. when the viscous forces strongly contribute to the friction. By investigating laser-induced bubbles on gold and yttrium iron garnet targets as a function of the liquid viscosity, using a nanosecond laser and an ultrafast shadowgraph imaging setup, we give a deeper insight into what determines the bubble dynamics. We find that the competition between the viscous forces and the surface tension (capillary number $\mathrm{Ca}$ ), on the one hand, and the competition between the viscous forces and inertia (Reynolds Number $R e$ ), on the other hand, are both key factors. Increasing the viscous forces and hereby $\mathrm{Ca}$ up to 100 has an impact on the bubble shape and result in a very pronounced rim, which separates the bubble in a spherical cap driven by inertia and an interlayer. The temporal evolution of the footprint radius of the interlayer can be addressed in the framework of the inertiocapillary regime. For an intermediate viscosity, the thickness of the interlayer is consistent with a boundary layer equation. Interestingly, our data cannot be interpreted with simplified hydrodynamic (Cox-Voinov) or molecularkinetic theory models, highlighting the originality of the dynamics reported when extremely high capillary numbers are achieved. Upon bubble collapse, spherical persistent microbubbles are created and partly dispersed in water, whereas the high-viscous polyalphaolefines lead to long-standing oblate persistent bubbles sticking to the target's surface, independent of the ablated target. Overall, liquid's viscosity determines laser ablation-induced cavitation.
\end{abstract}

\section{Keywords}

Laser ablation in Liquids; oil; gold; yttrium iron garnet; contact angle; bubble shape;

\section{Introduction}


Producing colloids through laser ablation in liquid (LAL) has become a popular technique for its variety of accessible materials ${ }^{1-5}$. In contrast to chemical routes, LAL works without using surfactants or chemical precursors in a one-step process ${ }^{6}$ with a wide variety of liquids ${ }^{7-14}$. Although LAL is scalable ${ }^{15}$, economically feasible ${ }^{16}$ and has a high potential for applications in biomedicine ${ }^{17,18}$, catalysis ${ }^{2,19}$, optics $^{20-22}$, additive manufacturing ${ }^{23,24}$ and nanoparticle-polymer composites ${ }^{25,26}$, there is still a lack of understanding for the basic mechanism involved during nanoparticle synthesis. The dynamics after the impact of a short laser pulse on a target in a liquid environment can be separated into the following stages. An early laser-induced plasma is quickly quenched a few microseconds after the impact, leading to a vapour bubble ${ }^{27-32}$ containing nanoparticles ${ }^{33-36}$. The bubble grows and collapses for first within a few hundreds of microseconds and finally leads to the release of the nanoparticles into the liquid ${ }^{34,35}$ when the bubble fully collapses and disappears after a few oscillations ${ }^{26,37-39}$. Persistent microbubbles are released during the collapse phase, which may shield a large portion of subsequent laser pulse in particular at high liquid viscosities ${ }^{40}$, and consist of chemical reaction products of the solvent and target, such as volatile carbohydrates ${ }^{40}$, hydrogen, oxygen and hydrogen peroxide ${ }^{41}$.

The dynamics of the cavitation bubble and its influence on the nanoparticle formation has been investigated by many researchers ${ }^{28,37-39,42-44}$. Surprisingly, there is a lack of literature investigating cavitation bubbles in highly viscous liquids, although viscous liquids are becoming more and more interesting for LAL applications in oils ${ }^{45,46}$ or monomers ${ }^{47}$ through offering the possibility for one-step synthesis of colloids. For example, nanoparticles as additives in engine oil are relevant for the automotive industry, where nanoparticles are often used to enhance the tribological properties of the lubricant ${ }^{46,48}$. The one-step synthesis via LAL could replace the process of dispersing chemical synthesised particles in oils which often leads to agglomeration and thus to lower functionality. However, it is still mostly unknown how viscosity and surface tension influence shape, lifetime and size of the laser-generated bubble, and how they influence the nanoparticle growth and agglomeration inside the bubble ${ }^{49,50}$. A deeper understanding could offer new ways to ultimately improve the overall LAL process, i.e. repeatability and productivity. A perfect hemispherical bubble is expected in an inviscid flow ${ }^{51}$, and most of the above-mentioned studies on laser ablation in low-viscosity liquids (water, ethanol ...) assume a hemispherical shape. Only a few reports deal with the complete description of the bubble shape (rim, interlayer, contact angle). Tomko et al. reported an asymmetric shrinking ${ }^{35}$. Contact angle hysteresis and bell-shaped bubbles in water have been observed ${ }^{39}$. Ibrahimkutty et al. reported an inward jet ${ }^{36}$ which agrees with a numerical study by Lechner et al. using finite-volume method applied to Navier-Stokes equations for a compressible fluid ${ }^{51,52}$. From studies of cavitation bubbles close to a solid-liquid interface it is further known that the viscosity has a significant influence on the bubble shape ${ }^{53}$. Furthermore, the very fast and thin jet observed in the cavitation-collapse-simulations by Lechner et al. is unlikely to be observed at liquid viscosity 40 times 
higher than water as the annular inflow is no longer fast enough. At such high viscosities, a jet forms which is much wider and much slower (on the order of $100 \mathrm{~m} / \mathrm{s}$ ) as in water (about $1000 \mathrm{~m} / \mathrm{s}$ ) [personal communication with Christiane Lechner]. Still, there is no model available yet to fully describe the shape of the LAL-induced bubbles for any viscosity. Moreover, laser-induced bubbles close to a surface are characterised by fast moving vapour-liquid interface. By experimentally investigating the bubble dynamics in viscous liquids, we could get access to an unusual high value for the capillary number and address original and unprecedented condition in the literature. In this study, we investigate the relevant parameters (e.g. bubble size, velocity, lifetime, contact angle, contact area) for laser ablation of both gold and yttrium-iron-garnet (YIG) in water and in highly viscous polyalphaolefin (PAO), and we take a deeper look on the origin of the deviations from the semi-spherical shape.

\section{Experimental}

The experiments were carried out in commercially available Spectrasync PAO6 (density $\rho=0.827 \mathrm{~g} / \mathrm{cm}^{3}$, kinematic viscosity $v=80.8 \mathrm{~mm}^{2} / \mathrm{s} @ 293^{\circ} \mathrm{K}$ ) and Spectrasync PAO40 (density $\rho=0.850 \mathrm{~g} / \mathrm{cm}^{3}$, kinematic viscosity $v=764 \mathrm{~mm}^{2} / \mathrm{s} @ 293^{\circ} \mathrm{K}$ ) supplied by Exxon Mobile. The viscosity of PAO is influenced by its chain length. Pure water $\left(18.2 \mathrm{M} \Omega / \mathrm{cm}^{2}\right)$ was used as a reference fluid (density $\rho=0.997 \mathrm{~g} / \mathrm{cm}^{3}$, kinematic viscosity $v=1.00 \mathrm{~mm}^{2} / \mathrm{s} @ 293^{\circ} \mathrm{K}$, surface tension $\left.\gamma=72.8 \mathrm{mN} / \mathrm{m}\right)$. The surface tension $\gamma$ also changes to $29.7 \mathrm{mN} / \mathrm{m}$ for PAO6 and $31.5 \mathrm{mN} / \mathrm{m}$ for PAO40 (@ 297 $\mathrm{K}$ ). As ablation targets we have chosen a commercially available gold foils $(99.99 \%)$ and yttrium-iron-garnet $\left(\mathrm{YIG}_{1} \mathrm{Y}_{3} \mathrm{Fe}_{5} \mathrm{O}_{12}\right)$ wafers to experience different wettability. The uncleaned gold foil is assumed more hydrophobic than the hydrophilic oxides. Note that the hydrophilicity does not only depend on the material, but can also be altered by impurities, microstructures, and adsorbed species on the surfaces ${ }^{54}$. The first bubble expansion and its collapse are very fast with respect to the heat conduction characteristic time. Therefore, the bubble growth is assumed to be adiabatic as it was shown by Lam et al. ${ }^{28}$ and we can neglect the heat transfer from the inner bubble to the liquid. As a result, we consider the kinematic viscosity and the surface tension constant during the first bubble expansion and collapse.

For the fast imaging shadowgraphy experiments, a target is placed in a cubic vessel with $6 \mathrm{ml}$ of liquid. The liquid layer thickness above the target is $6.5 \mathrm{~mm}$. For the sake of comparability, the liquid layer thickness above the target is fixed throughout all experiments. The third harmonic of a pulsed Nd-YAGlaser ( $355 \mathrm{~nm}, 5 \mathrm{~ns}, 9 \mathrm{~Hz}, 7.2 \mathrm{~mJ} /$ pulse) is focused on a spot with a diameter of approximately $260 \mu \mathrm{m}$, resulting in a fluence of $13.6 \mathrm{~J} / \mathrm{cm}^{2}$. In our setup geometry, the liquid's transmission at $355 \mathrm{~nm}$ for a $6.5 \mathrm{~mm}$ liquid thickness is $99 \%$ for water, $97 \%$ for PAO6, and 96\% for PAO40, ensuring similar fluence for the three liquids (see supplementary Fig. S1). Ablation is observed at 210000 frames per second, 
which corresponds to an integration time of $4.75 \mu \mathrm{s}$, by an ultra-fast camera (Phantom v711, Vision Research). On the camera, a Zoom 6000 from Navitar is mounted. The magnification of the optical system is 0.65 . According to its performance specification, the resolution limit of the Zoom 6000 optical assembly is then $31 \mu \mathrm{m}$, which also corresponds to the image of a single-pixel ( $20 \mu \mathrm{m}$ in size divided by the magnification). It leads to an overall resolution of $44 \mu \mathrm{m}(\sqrt{2} \times 31 \mu \mathrm{m})$. A pattern of ultra-bright LEDs is used for illumination. To avoid distorting the results by inhomogeneities of the target surface, or through changes in the liquid parameters causing changes in the target illumination, each experiment is performed on a fresh target spot in fresh liquid while the vessel is cleaned between each experiment. The camera is synchronised with a laser beam shutter to ensure that the camera starts imaging right after the first laser shot on a fresh surface.

\section{Results}

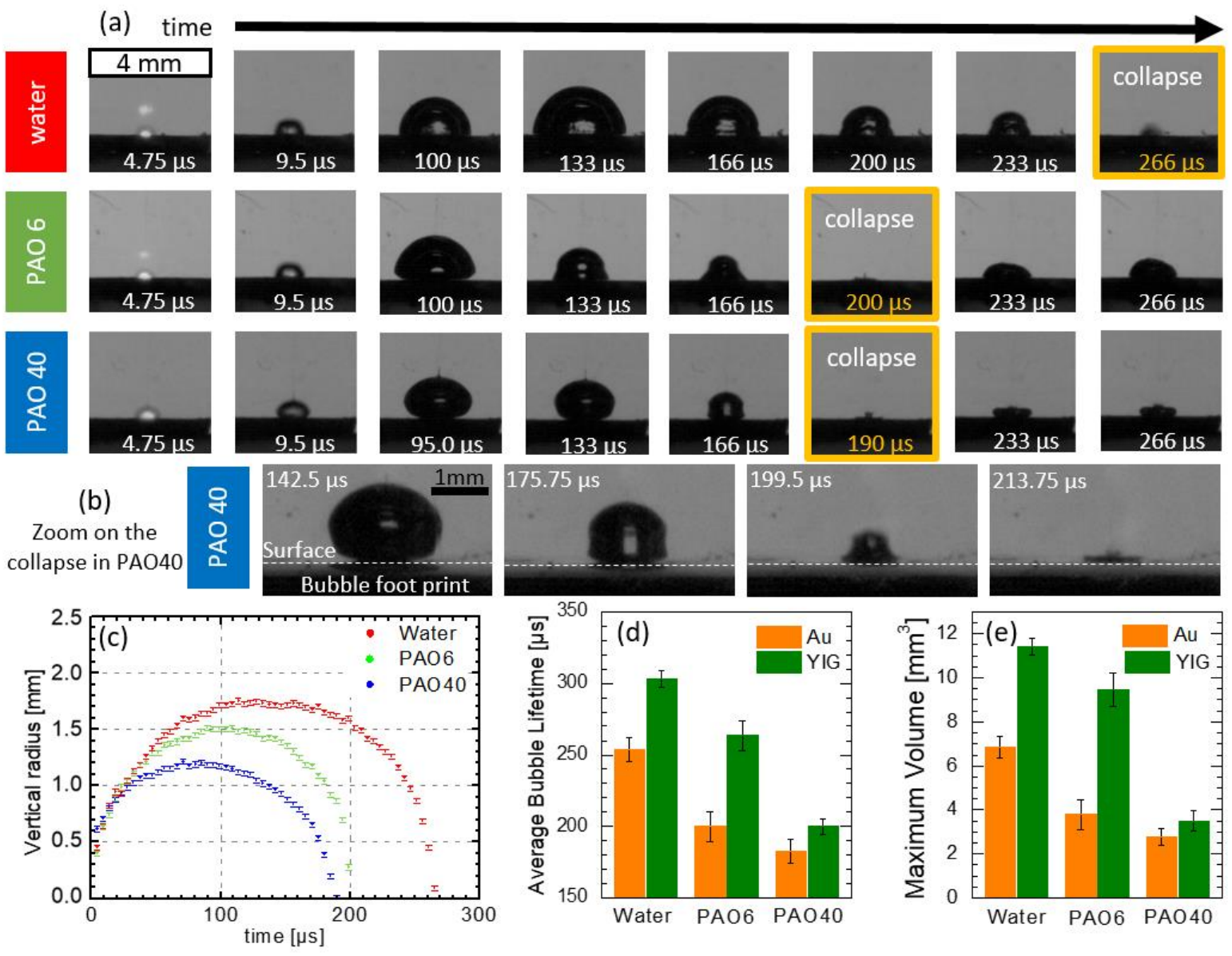

Fig. 1: (a) Shadowgrams of the cavitation bubbles after the impact of a ns-laser pulse on a gold target in water (top), PAO6 (middle) and PAO40 (bottom). Videos of both gold and YIG LAL in water and PAOs are available in the supplementary materials. (b) Zoom on the collapse in PAO40 with a focus on the footprint diameter, as well as the persistent oblate bubble after oscillation. (c) Vertical radius (height) 
for each liquid plotted as a function of time. The error bars correspond to the resolution of the imaging system ( $\pm 22 \mu \mathrm{m}$ ). (d) Lifetime and (e) maximum volume of the first bubble for a gold target and an YIG target. The error bars correspond to the $80 \%$ confidence interval according to the Student's tdistribution.

Fig. 1a shows the dynamics of the bubbles in three liquids for a gold target (videos for both the gold and YIG targets are available in the supplementary materials). Starting with the first image $4.75 \mu$ s after the laser impact, the bubble starts to expand, reaching its maximum expansion after $100-150 \mu \mathrm{s}$. Interestingly, the bubbles in PAO6 and PAO40 continue expanding in the vertical direction, even after the expansion in the horizontal direction stopped, highlighting the strength of the friction. The footprint of the bubble in PAO40 stays almost constant during the collapse which could affect the redeposition of ablated material on the target surface (see Fig. 1b).

Fig. $1 \mathrm{c}$ depicts the vertical bubble radius (height) for the different liquids as a function of time. Assuming a rotational symmetry of the bubble shape, the maximum volume is deduced from the bubble shape at its maximum vertical radius (Fig. 1d). The average maximum volume for each liquid is shown in Fig. 1e. After the maximum expansion, the shrinking starts, ending with the collapse of the cavitation bubble followed by bubble oscillations (videos of the whole process including oscillation are provided in the supplementary information). The total lifetime of the first bubble is between 190 and $280 \mu \mathrm{s}$, depending on the liquid. For each bubble, the lifetime is consistent with the Rayleigh collapse time deduced from the maximum vertical radius (See supplementary Fig. S2). Compared to water, the number of oscillations is smaller in the PAOs. Obviously, the highly viscous PAOs provide higher damping of the bubble oscillation through viscous energy dissipation, resulting in only one oscillation for PAO40. After oscillations, persistent bubbles ${ }^{55}$ are formed from the cavitation bubble and their mobility decreases dramatically for high viscosities. The collapse in water releases microbubbles into the liquid that only partly adhere to the target surface. In contrary, LAL in PAO40 results in persistent oblate-shaped bubbles sticking to the target, independent of the ablated target material (see Fig. $2 \mathrm{c}$ and the videos in the supplementary information).

There are changes when increasing the viscosity of the liquid, not only in the lifetime and the maximal size of the bubble, but also in the shape and contact angle between the target and the bubble. The physics of spherical and hemispherical bubble oscillation has extensively been studied in the past ${ }^{56}$ with focus on bubbles in water. Various studies are also available for cavitation bubbles at different distances from a solid boundary ${ }^{51,57,58}$ To discuss the differences in the bubble dynamics in the present case, we have developed a Python code to deduce from the movie the time evolution of the relevant 
geometrical parameters characterizing the bubble shape. For each image, the bubble shape is fitted using a circular arc for the top of the bubble (spherical shape) and a continuous line for the interlayer (conical shape). The shape of the interlayer is assumed to be conical to measure the apparent contact angle $\theta$. The geometrical quantities deduced from the fit are the height of the bubble $R$, which remains almost equal to the radius of curvature during the bubble expansion, the footprint radius $X$, the distance $L$ between the bubble boundary (edge of the interlayer) and the centre of the bubble, the thickness $e$ of the interlayer, and the contact angle $\theta$ (see Fig. 2a-b).

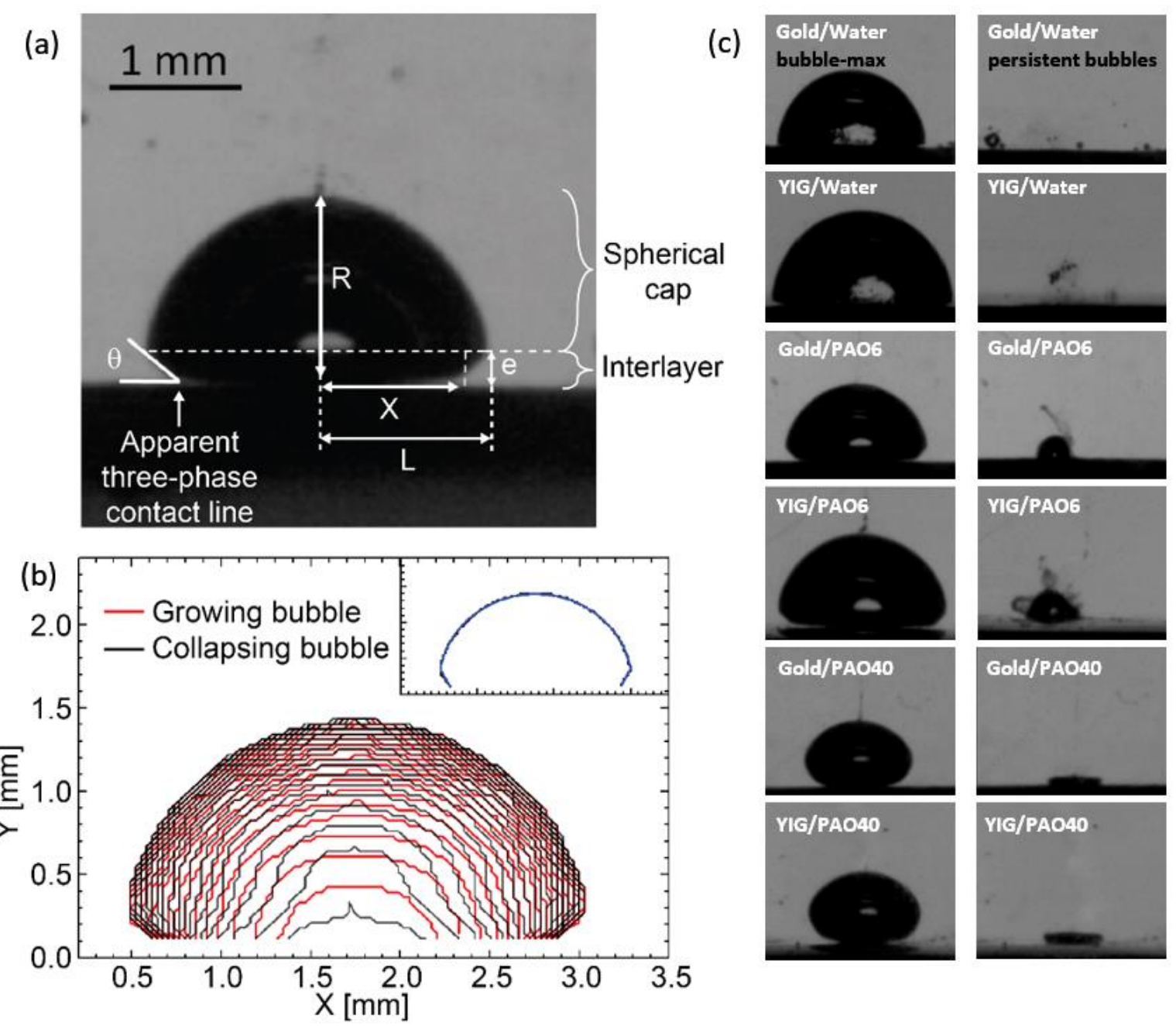

Fig. 2: (a) Image of a laser-generated bubble on gold in PAO6 showing the relevant geometrical parameters. (b) The red and black curves correspond to the bubble shape returned by the image processing (raw data) for the expanding and receding phases, respectively. Inset: the black curve is the bubble contour obtained from raw data and the blue curve corresponds to its fit. c) Images of the cavitation bubbles at their maximal expansion (left panel) and persistent bubbles after oscillation (right panel) depending on target (gold, YIG) and liquid (water, PAO6, PAO40) combinations. 
From the measurement of $R, \mathrm{X}$ and $L$ as a function of time, we can compute velocities:

$$
\mathrm{V}_{\mathrm{t}}=\frac{\mathrm{dR}}{\mathrm{dt}} ; \quad \mathrm{V}_{\mathrm{cl}}=\frac{\mathrm{dX}}{\mathrm{dt}} ; \quad \mathrm{V}_{\mathrm{e}}=\frac{\mathrm{dL}}{\mathrm{dt}}
$$

$V_{t}$ is the velocity of the vapour/liquid interface at the top of the bubble, $V_{c l}$ is the velocity of the apparent three-phase contact line, and $V_{\mathrm{e}}$ is the velocity of the vapour/liquid interface at the edge of the bubble.
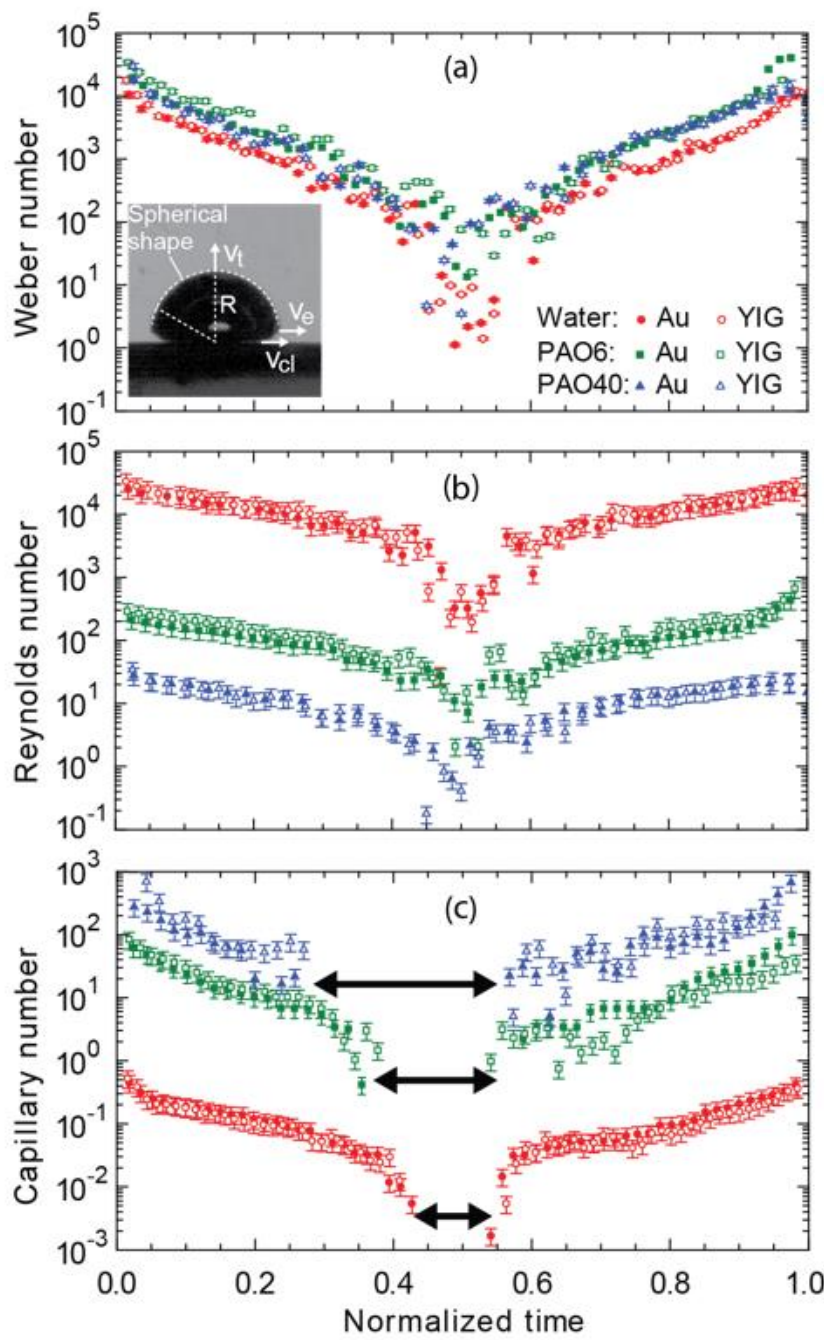

Fig. 3: (a) Weber number We and (b) Reynolds Re number calculated from the velocity $V_{\mathrm{t}}$ at the top of the bubble. (c) Capillary number $C a$ was calculated from the velocity $V_{\mathrm{cl}}$ of the apparent contact line. The normalized time corresponds to the duration of the first expansion and shrinking of the bubble. No movement is observed $\left(V_{\mathrm{cl}}=0\right)$ for the apparent contact line during the period delimited by the arrows. Error bars are deduced from the propagation of uncertainty (see supplementary) and take into account an uncertainty of $\pm 30 \%$ on the viscosity, an uncertainty of $\pm 3 \%$ on the surface tension ${ }^{59,60}$, and an uncertainty of $\pm 22 \mu \mathrm{m}$ on the geometrical parameters ( $R$ and $X$ ). 
Fig. 3 (a-b) show the Reynolds number Re and the Weber number We computed from the velocity $V_{\mathrm{t}}$ of the top of the bubble, while figure 3 (c) shows the capillary number $C a$ computed from the velocity $V_{c l}$ of the apparent contact line.

$$
\begin{gathered}
W e=\rho V_{t}^{2} R / \gamma \\
R e=V_{t} R / v \\
C a=\rho \nu V_{\mathrm{cl}} / \gamma
\end{gathered}
$$

$\rho$ is the fluid mass density, $\gamma$ the surface tension, and $v$ the kinematic viscosity. $R$ is the height of the bubble, which remains almost equal to the radius of curvature of the cap during bubble expansion. $R e$ and We values do not appear to significantly depend on the target material. Re and We mainly scale with the kinematic viscosity $\left(\propto v^{-1}\right)$ and the surface tension $\left(\propto \gamma^{-1}\right)$, respectively, because the velocity $V_{\mathrm{t}}$ and the height $R$ of the bubbles do not differ significantly from a liquid to another (for a given normalized time, less than one order of magnitude). As Re and $\mathrm{Ca}$ depend on the kinematic viscosity, three regimes are clearly distinguishable, on the opposite of We evolution which only deals with surface tension. High values of We and Re are achieved for the three solvents, which indicate that the balance between inertia and inner pressure dominates the motion of the bubbles cap. The three regimes then mainly concern the bubble motion close to the surface. By comparing viscous force with inertia, Re shows decreasing values with viscosity. From water to PAO6 and PAO40, Re is reduced by 2 and 3 orders of magnitude, respectively, and is close to unity for the more viscous oil. This trend is confirmed by the evolution of $\mathrm{Ca}$. In water, $\mathrm{Ca}$ is less than unity in the first $\mu$ s before reaching $10^{-2}$ at the maximum bubble size. In PAO6 and PAO40, the same evolution is shifted by 2 and 3 orders of magnitude towards high capillary numbers. Contrary to the case of water, the viscous force cannot be neglected anymore with respect to inertia in polyolefin. The relatively high polyolefin viscosity leads to contact-line friction ${ }^{61}$ which competes with inertia and then drives the bubble motion close to the surface.

The main differences in the bubble dynamics between different viscosities can be observed in the behaviour of the interlayer defined in fig 2 . The spreading of the bubble on the solid target can be compared to the advancing and receding sessile droplet ${ }^{62-64}$ or a spreading bubble ${ }^{65,66}$. During the early stage of bubble expansion (first quarter of the bubble lifetime), the time-dependent footprint radius $X(t)$ of the bubble follows the power-law $X(t) \propto t^{n}$ (see supplementary Fig. S3 and S4). The corresponding exponent $\mathrm{n}$ which characterizes the spreading regimes is shown in Table 1 and indicates 
only a weak dependence of the bubble expansion on the target material. The exponent $\mathrm{n}$ is around 0.39 for water. Such value is consistent with regimes where the dominant resistance is liquid inertia. For a bubble characterized by a constant volume, these regimes lead to theoretical time evolution between $t^{1 / 2}$ and $t^{1 / 365}$ depending on the driving force, the gravity or the capillarity, respectively, which counterbalances the inertia. If the bubble size is larger or smaller than the capillary length, this leads to a flattened or a spherical shape, respectively. By considering our bubble shape as hemispherical, an inertiocapillary regime with $t^{1 / 3}$ is in good agreement with the data in Table 1 for water and PAO6. However, for laser-generated bubbles, the driving force during the early expansion of the bubble is clearly different and corresponds to the bubble inner pressure. Moreover, the bubble volume is not constant. In water, for large Weber and Reynolds numbers, a simplified Rayleigh-Plesset equation applies and reflects the balance between inertia and inner pressure $\left(P_{B}\right)^{28}$ :

$$
\rho\left(R \ddot{R}+\frac{3}{2} \dot{R}^{2}\right)=P_{B}(t)-P_{l}
$$

where $P_{l}$ denotes the surrounding liquid pressure $\left(<<P_{B}\right)$, and the inner pressure $P_{B}$ follows the isentropic relation $P_{B} \propto A R^{-3 \alpha}$ with $\alpha$ the heat capacity ratio and a constant $A{ }^{28}$. It leads to the balance $\rho \frac{R^{2}}{t^{2}}=A R^{-3 \alpha}$, and then to the power law coefficient $n=\frac{2}{3 \alpha+2}$. For a laser-generated bubble, we can expect $n=1 / 3$ for water ( $\alpha_{\text {water }}=1.33$ ) and $n=2 / 5$ for large molecules ( $\alpha$ tends to 1 ). These values are consistent with the measured ones for water $(X(t)$ and $R(t)$ are identical for water) and PAO6. In contrast, $n$ is significantly smaller for the most viscous PAO40 $(n \approx 0.2)$ which indicates a change of regime to domination of viscous forces. By increasing the viscosity, the Reynolds number decreases, and the Capillary number drastically increases. Close to the surface, friction has to be included in the resistance forces along with inertia. For a droplet characterized by a constant volume, when inertia is marginal, the balance between the surface tension and the viscous dissipation leading to friction at small scale is described by Tanner's law with $t^{1 / 10}$. A decrease of the $n$ value with increasing viscosity makes sense, even if Tanner's law assumes again a constant volume, as well as a low capillary number $(C a<<1)$, which only applies for the bubble's dynamics close to their maximum radius (see figure 3 ). To our best knowledge, there is no study available in the literature describing a droplet or bubble spreading in a high capillary number regime such the ones reached for high viscous PAOs, i.e. up to 100. However, large Ca numbers are achieved in the context of high-speed coating, up to 1000 for the coating of optical fibres (see ${ }^{67,68}$ and references inside).

Table 1: Value of the parameter $\mathrm{n}$ from the fit with $\mathrm{X}(t) \propto t^{n}$, where $X(t)$ is the time-dependent of the footprint radius of the bubble during its early expansion, i.e. the first quarter of the bubble lifetime 
(see supplementary Fig. S3 and S4). The values in brackets correspond to the $80 \%$ confidence interval according to the Student's t-distribution.

\begin{tabular}{|c|c|c|}
\hline$n$ & Gold & YIG \\
\hline Water & $0.389( \pm 0.051)$ & $0.393( \pm 0.028)$ \\
\hline PA06 & $0.332( \pm 0.073)$ & $0.334( \pm 0.05)$ \\
\hline PA040 & $0.194( \pm 0.035)$ & $0.200( \pm 0.053)$ \\
\hline
\end{tabular}

The velocity of the apparent three-phase contact line is drawn as a function of the apparent dynamic contact angle in Fig. 4 for PAO6, PAO40 and water. In the case of water, the expanding bubble is hemispherical, and the apparent contact angle is constant around $90^{\circ}$ during most of the bubble lifetime (also see Fig. 1a) due to the inertial regime with a low capillary number (see Fig. 3c). There is only a deviation from $90^{\circ}$ during the collapse phase (see Fig. 4) characterized by a sharp acceleration of the bubble. When increasing the liquid viscosity and hereby the capillary number $\mathrm{Ca}$ by several orders of magnitude (see Fig. 3c), we observe the appearance of hysteresis between expanding and shrinking bubble for the apparent contact angle between target and bubble. The hysteresis is similar to the behaviour observed for a moving liquid droplet or a dynamic sessile drop at small Ca numbers $63,69,70$. In the limit of the small Ca number, the apparent contact angle is described by the Cox-Voinov hydrodynamic model of dynamic wetting ${ }^{71}$ and has been extensively studied ${ }^{63,69}$. But this relation does not apply here. A molecular-kinetic theory has been also developed to describe the velocitydependence of the dynamic contact ${ }^{58,61}$ Blake et al. introduced activation energies of adsorption and desorption from the surface of the molecules of the liquid. The velocity-dependence of the dynamic contact angle is then due to the perturbation of the adsorption equilibrium due to motion of the contact line. Yet again, the model didn't succeed to fit the data for the largest capillary numbers, highlighting the originality of the dynamics reported here.

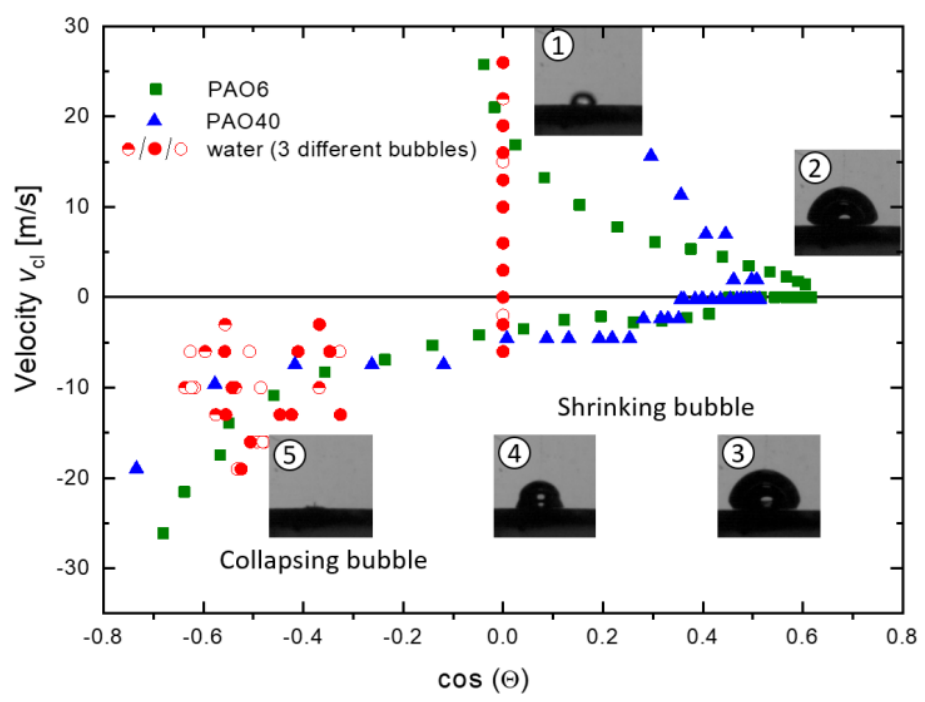


Fig. 4: Velocity of the apparent contact line as a function of the apparent contact angle plotted for a few laser-induced bubbles on a gold target in PAO6 (green squares), PAO40 (blue triangles) and in water (red circles). Pictures correspond to a laser-generated bubble in PAO6.

The interlayer does not only come with a time-dependent angle, but also with a time-dependent height. Fig. 5 (a) shows the time-dependent height of the interlayer during the expansion for the two targets in PAO6. The error bars are based on the calculated temporal and special resolution of the experimental setup. The interlayer for PAO40 is not addressed because the round shape of the bubble would lead to an arbitrary definition of the parameters $L$ and $e$. For PAO6, Fig. 5 (a) shows no differences in the height of the boundary layer between a gold and an oxide target. The thickness of the interlayer increases with time and fits the following boundary layer equation with the dimensionless constant $Z_{\mathrm{b}}$ :

$$
e=Z_{b} \sqrt{\frac{v \mathrm{~L}}{V_{e}}}
$$

$V_{e}=d L / d t$ is the velocity of the liquid/vapour interface at the edge of the bubble, $v$ is the kinematic viscosity and $L$ is the distance between the bubble boundary at the edge of the interlayer and the centre of the bubble. The slope of the regression lines in Fig. 5 (b) represent the dimensionless constant

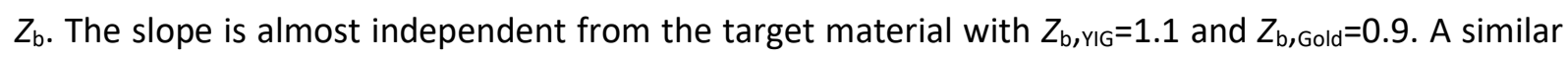
behaviour has been observed by Van Ouwerkerk for bubbles on a hot surface during boiling of the liquid ${ }^{72,73}$. Assuming no change in the shape during the bubble growth, Van Ouwerkerk deduced $Z_{b}=0.9$ from a self-similar approach and reported experimental values for $Z_{\mathrm{b}}$ between 0.3 and 0.8 .
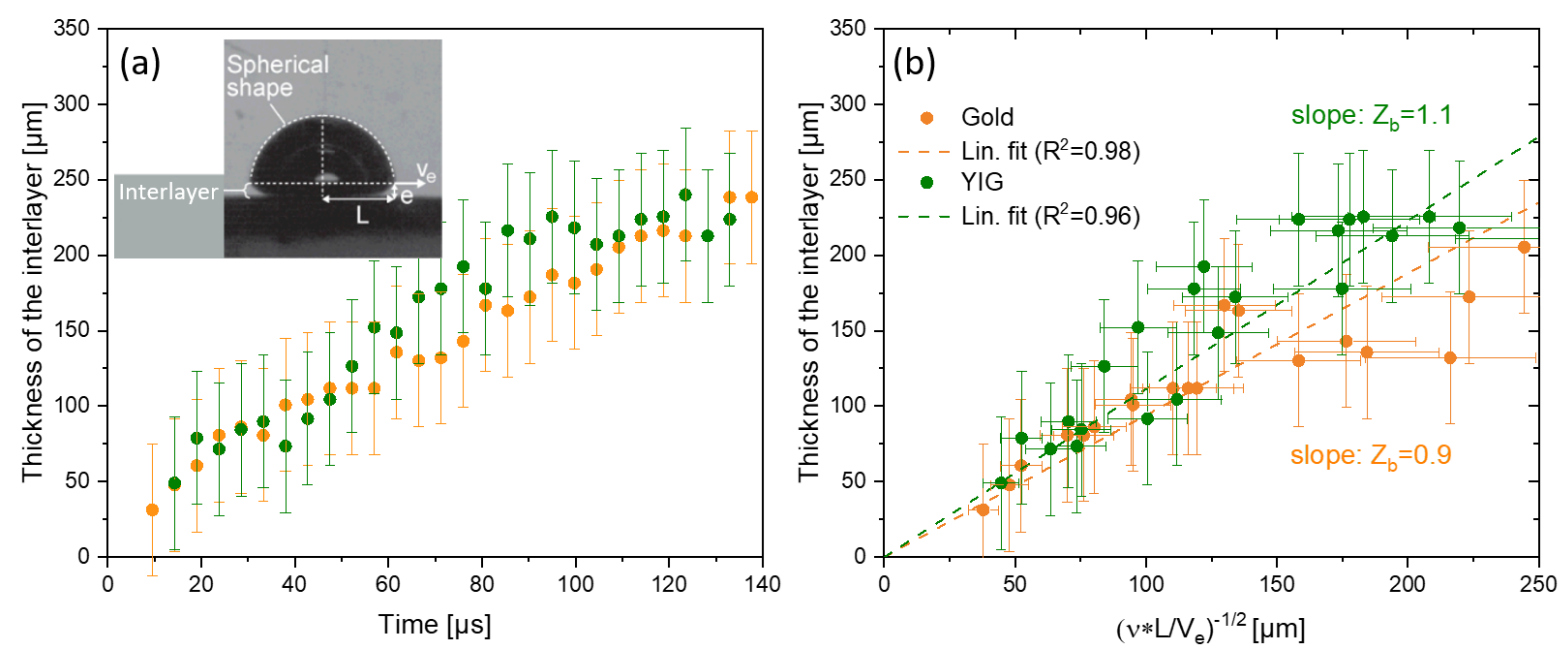

Fig. 5: Measured thickness e of the interlayer of bubbles on gold and YIG in PAO6 during the expansion phase of the bubble (a) as a function of time and (b) as a function of the ratio $\sqrt{v * L / V_{e}}$ with the 
slope $Z_{\mathrm{b}}$ as a proportionality factor (the inset shows the definition of the interlayer). The coefficient of determination is $R^{2}=0.96$ for YIG and $R^{2}=0.98$ for gold. The error bars for the layer thickness are based on the optical resolution (see experimental section) and the $\mathrm{x}$-axis error is estimated following the propagation of uncertainty.

\section{Conclusion}

Laser-induced cavitation in liquids is a key method applied to understand cavitation near solid boundaries, but also highly relevant for laser synthesis of colloids. Indeed, the cavitation bubble but also the resulting persistent microbubbles may shield subsequent laser pulses, affecting productivity and reproducibility of the laser generation of nanoparticles. While it is intuitive that viscosity plays a role, experimental investigations under variation of both the target and liquid types have been missing. In this work we showed that an increase of the kinematic viscosity by 2 orders of magnitude compared to water does affect the lifetime, the shape, the size and the damping of the oscillation of laser-induced cavitation bubbles. Bubbles can be divided into two geometrical sections, separated by a rim: A spherical cap with a dynamic driven by inertial forces and an interlayer close to the target where the shape differs from a sphere when the contribution of the viscous forces to the friction drastically increases. Indeed, looking at the bubble footprint dynamics close the target, an inertiocapillary regime is observed for intermediate viscosity and the thickness of the interlayer is in accordance with a boundary layer equation. Moreover, a contact angle hysteresis appears with increasing viscosity. These findings poorly depend on the target material which suggests that the shape and the dynamics of the bubble are driven by the contribution of the viscous forces to the friction, rather than by static hydrophilicity of the target. However, by increasing the viscosity, the behaviour of the contact line cannot be interpreted with simplified hydrodynamic (Cox-Voinov) or molecular-kinetic theory models, highlighting the originality of the dynamics reported when extremely high capillary numbers are achieved. In addition to viscosity effects on the first cavitation of laser-induced bubbles, persistent bubbles show noteworthy phenomenological differences caused by the liquid and appear independent of the target type. These bubbles are spherical and partly dispersed for laser ablation in water, whereas in PAO40 a larger, flat, persistent bubble adheres to the target.

\section{Supplementary Material}

See supplementary material for more information on bubble height, bubble shrinking and the automated data processing for shadowgraph imaging. The supplementary material also provides information on the time dependence of the footprint radius and on the Rayleigh collapse time. 


\section{Acknowledgement}

This work has been financially supported by the European Cooperation in Science and Technology through COST Action MP1302 Nanospectroscopy. Tim Hupfeld thanks Evonik Industries for financial support. We would like to thank Carsten Dubs from INNOVENT e.V. Technologieentwicklung for providing the YIG wafer. BG and SB thank the Deutsche Forschungsgemeinschaft DFG for funding.

\section{References}

${ }^{1}$ D. Zhang, B. Gökce, and S. Barcikowski, Chem. Rev. 117, 3990 (2017).

${ }^{2}$ D. Zhang, J. Liu, P. Li, Z. Tian, and C. Liang, ChemNanoMat 3, 512 (2017).

${ }^{3}$ V. Amendola and M. Meneghetti, Phys. Chem. Chem. Phys. 11, 3805 (2009).

${ }^{4}$ J. Xiao, P. Liu, C.X. Wang, and G.W. Yang, Prog. Mater. Sci. 87, 140 (2017).

${ }^{5}$ D. Amans, W. Cai, and S. Barcikowski, Appl. Surf. Sci. 488, 445 (2019).

${ }^{6}$ J.G. Walter, S. Petersen, F. Stahl, T. Scheper, and S. Barcikowski, J. Nanobiotechnology 8, 21 (2010).

${ }^{7}$ V. Amendola, S. Polizzi, and M. Meneghetti, Langmuir 23, 6766 (2007).

${ }^{8}$ H. Wender, M.L. Andreazza, R.R.B. Correia, S.R. Teixeira, and J. Dupont, Nanoscale 3, 1240 (2011).

${ }^{9}$ H.P.S. Castro, V.S. Souza, J.D. Scholten, J.H. Dias, J.A. Fernandes, F.S. Rodembusch, R. Dos Reis, J. Dupont, S.R. Teixeira, and R.R.B. Correia, Chem. - A Eur. J. 22, 138 (2016).

${ }^{10}$ G. Compagnini, A.A. Scalisi, and O. Puglisi, Phys. Chem. Chem. Phys. 4, 2787 (2002).

${ }^{11}$ N. Takada, T. Sasaki, and K. Sasaki, Appl. Phys. A Mater. Sci. Process. 93, 833 (2008).

${ }^{12}$ N. Tabatabaie and D. Dorranian, Appl. Phys. A 122, 558 (2016).

${ }^{13}$ T. Kato, S. Stauss, S. Kato, K. Urabe, M. Baba, T. Suemoto, and K. Terashima, Appl. Phys. Lett. 101, 224103 (2012).

${ }^{14}$ E.B. Gordon, A. V. Karabulin, V.I. Matyushenko, V.D. Sizov, and I.I. Khodos, J. Low Temp. Phys. 172, 94 (2013).

${ }^{15}$ R. Streubel, G. Bendt, and B. Gökce, Nanotechnology 27, 205602 (2016).

${ }^{16}$ S. Jendrzej, B. Gökce, M. Epple, and S. Barcikowski, ChemPhysChem 18, 1012 (2017).

${ }^{17}$ N. Luo, X. Tian, J. Xiao, W. Hu, C. Yang, L. Li, and D. Chen, J. Appl. Phys. 113, 164306 (2013).

${ }^{18}$ C. Streich, L. Akkari, C. Decker, J. Bormann, C. Rehbock, A. Müller-Schiffmann, F.C. Niemeyer, L. Nagel-Steger, D. Willbold, B. Sacca, C. Korth, T. Schrader, and S. Barcikowski, ACS Nano 10, 7582 (2016).

${ }^{19}$ J. Zhang, M. Chaker, and D. Ma, J. Colloid Interface Sci. 489, 138 (2017).

${ }^{20}$ J. Liu, X. Tian, N. Luo, C. Yang, J. Xiao, Y. Shao, X. Chen, G. Yang, D. Chen, and L. Li, Langmuir 30, 13005 (2014).

${ }^{21}$ C. Ma, J. Yan, Y. Huang, and G. Yang, Adv. Opt. Mater. 5, 1700761 (2017).

${ }^{22}$ V. Amendola, S. Scaramuzza, S. Agnoli, G. Granozzi, M. Meneghetti, G. Campo, V. Bonanni, F. Pineider, C. Sangregorio, P. Ghigna, S. Polizzi, P. Riello, S. Fiameni, and L. Nodari, Nano Res. 8, 4007 (2015). 
${ }^{23}$ T. Hupfeld, T. Laumer, T. Stichel, T. Schuffenhauer, J. Heberle, M. Schmidt, S. Barcikowski, and B. Gökce, Procedia CIRP 74, 244 (2018).

${ }^{24}$ C. Doñate-Buendía, F. Frömel, M.B. Wilms, R. Streubel, J. Tenkamp, T. Hupfeld, M. Nachev, E. Gökce, A. Weisheit, S. Barcikowski, F. Walther, J.H. Schleifenbaum, and B. Gökce, Mater. Des. 154, 360 (2018).

${ }^{25}$ D. Longano, N. Ditaranto, N. Cioffi, F. Di Niso, T. Sibillano, A. Ancona, A. Conte, M.A. Del Nobile, L. Sabbatini, and L. Torsi, Anal. Bioanal. Chem. 403, 1179 (2012).

${ }^{26}$ N. Lasemi, U. Pacher, L. V. Zhigilei, O. Bomatí-Miguel, R. Lahoz, and W. Kautek, Appl. Surf. Sci. 433, 772 (2018).

${ }^{27}$ D. Amans, M. Diouf, J. Lam, G. Ledoux, and C. Dujardin, J. Colloid Interface Sci. 489, 114 (2017).

28 J. Lam, J. Lombard, C. Dujardin, G. Ledoux, S. Merabia, and D. Amans, Appl. Phys. Lett. 108, 074104 (2016).

${ }^{29}$ A. Letzel, B. Gökce, P. Wagener, S. Ibrahimkutty, A. Menzel, A. Plech, and S. Barcikowski, J. Phys. Chem. C 121, 5356 (2017).

${ }^{30}$ M. Dell'Aglio, R. Gaudiuso, O. De Pascale, and A. De Giacomo, Appl. Surf. Sci. 348, 4 (2015).

${ }^{31}$ M. Tiberi, A. Simonelli, G. Cristoforetti, P. Marsili, F. Giammanco, and E. Giorgetti, Appl. Phys. A Mater. Sci. Process. 110, 857 (2013).

${ }^{32}$ A. Tamura, A. Matsumoto, K. Fukami, N. Nishi, and T. Sakka, J. Appl. Phys. 117, 173304 (2015).

${ }^{33}$ C.Y. Shih, R. Streubel, J. Heberle, A. Letzel, M. V. Shugaev, C. Wu, M. Schmidt, B. Gökce, S. Barcikowski, and L. V. Zhigilei, Nanoscale 10, 6900 (2018).

${ }^{34}$ S. Ibrahimkutty, P. Wagener, A. Menzel, A. Plech, and S. Barcikowski, Appl. Phys. Lett. 101, 103104 (2012).

${ }^{35}$ J. Tomko, J.J. Naddeo, R. Jimenez, Y. Tan, M. Steiner, J.M. Fitz-Gerald, D.M. Bubb, and S.M. O'Malley, Phys. Chem. Chem. Phys. 17, 16327 (2015).

${ }^{36}$ S. Ibrahimkutty, P. Wagener, T.D.S. Rolo, D. Karpov, A. Menzel, T. Baumbach, S. Barcikowski, and A. Plech, Sci. Rep. 5, 16313 (2015).

${ }^{37}$ S. Reich, P. Schönfeld, P. Wagener, A. Letzel, S. Ibrahimkutty, B. Gökce, S. Barcikowski, A. Menzel, T. dos Santos Rolo, and A. Plech, J. Colloid Interface Sci. 489, 106 (2017).

${ }^{38}$ R. Tanabe, T.T.P. Nguyen, T. Sugiura, and Y. Ito, Appl. Surf. Sci. 351, 327 (2015).

${ }^{39}$ N. Dabir-Moghaddam, Z. Liu, and B. Wu, J. Appl. Phys. 121, 044908 (2017).

${ }^{40}$ M.-R. Kalus, N. Bärsch, R. Streubel, E. Gökce, S. Barcikowski, and B. Gökce, Phys. Chem. Chem. Phys. (2017).

${ }^{41}$ M.-R. Kalus, R. Lanyumba, N. Lorenzo-Parodi, M.A. Jochmann, K. Kerpen, U. Hagemann, T.C. Schmidt, S. Barcikowski, and B. Gökce, Phys. Chem. Chem. Phys. 21, 18636 (2019).

${ }^{42}$ K. Sasaki, T. Nakano, W. Soliman, and N. Takada, Appl. Phys. Express 2, 046501 (2009).

${ }^{43}$ A. De Giacomo, M. Dell'Aglio, A. Santagata, R. Gaudiuso, O. De Pascale, P. Wagener, G.C. Messina, G. Compagnini, and S. Barcikowski, Phys. Chem. Chem. Phys. 15, 3083 (2013).

${ }^{44}$ N. Takada, A. Fujikawa, and K. Sasaki, Jpn. J. Appl. Phys. 50, 126201 (2011).

${ }^{45}$ S. Jendrzej, B. Gökce, and S. Barcikowski, Chem. Eng. Technol. 40, 1569 (2017).

${ }^{46}$ M. Zhang, X. Wang, X. Fu, and Y. Xia, Tribol. Int. 42, 1029 (2009). 
${ }^{47}$ A. Menéndez-Manjón, A. Schwenke, T. Steinke, M. Meyer, U. Giese, P. Wagener, and S. Barcikowski, Appl. Phys. A Mater. Sci. Process. 110, 343 (2013).

${ }^{48}$ R. Chou, A.H. Battez, J.J. Cabello, J.L. Viesca, A. Osorio, and A. Sagastume, Tribol. Int. 43, 2327 (2010).

49 J. Tomko, S.M.O. Malley, C. Trout, J.J. Naddeo, R. Jimenez, J.C. Griepenburg, W. Soliman, and D.M. Bubb, Colloids Surfaces A Physicochem. Eng. Asp. 522, 368 (2017).

${ }^{50}$ T. Tsuji, D.-H. Thang, Y. Okazaki, M. Nakanishi, Y. Tsuboi, and M. Tsuji, Appl. Surf. Sci. 254, 5224 (2008).

${ }^{51}$ C. Lechner, W. Lauterborn, M. Koch, and R. Mettin, Phys. Rev. Fluids 4, 021601 (2019).

${ }^{52}$ M. Koch, C. Lechner, F. Reuter, K. Köhler, R. Mettin, and W. Lauterborn, Comput. Fluids 126, 71 (2016).

${ }^{53}$ S.R. Gonzalez Avila, C. Song, and C.D. Ohl, J. Fluid Mech. 767, 31 (2015).

${ }^{54}$ J. Drelich, E. Chibowski, D.D. Meng, and K. Terpilowski, Soft Matter 7, 9804 (2011).

${ }^{55}$ M. Kalus, N. Bärsch, R. Streubel, E. Gökce, S. Barcikowski, and B. Gökce, Phys. Chem. Chem. Phys. 19, 7112 (2017).

${ }^{56}$ W. Lauterborn and T. Kurz, Reports Prog. Phys. 73, 106501 (2010).

${ }^{57}$ L. Van Wijngaarden, Ultrason. Sonochem. 29, 524 (2016).

${ }^{58}$ C.F. Naudé and A.T. Ellis, J. Basic Eng. 83, 648 (1961).

${ }^{59}$ W. V. Kayser, J. Colloid Interface Sci. 56, 622 (1976).

${ }^{60}$ P. Pötschke, J. Pionteck, and H. Stutz, Polymer (Guildf). 43, 6965 (2002).

${ }^{61}$ E. Bertrand, T.D. Blake, and J. De Coninck, Colloids Surfaces A Physicochem. Eng. Asp. 369, 141 (2010).

${ }^{62}$ B. Lavi and A. Marmur, Colloids Surfaces A Physicochem. Eng. Asp. 250, 409 (2004).

${ }^{63}$ D. Bonn, J. Eggers, J. Indekeu, and J. Meunier, Rev. Mod. Phys. 81, 739 (2009).

${ }^{64}$ S.F. Toosi, S. Moradi, and S. Hatzikiriakos, Rev. Adhes. Adhes. 5, 55 (2017).

${ }^{65}$ H. De Maleprade, C. Clanet, and D. Quéré, Phys. Rev. Lett. 117, 1 (2016).

${ }^{66}$ J.D. Paulsen, R. Carmigniani, A. Kannan, J.C. Burton, and S.R. Nagel, Nat. Commun. 5, 3182 (2014).

${ }^{67}$ D. Jacqmin, J. Fluid Mech. 455, 347 (2002).

${ }^{68}$ T.D. Blake, R.A. Dobson, and K.J. Ruschak, J. Colloid Interface Sci. 279, 198 (2004).

${ }^{69}$ J.H. Snoeijer and B. Andreotti, Annu. Rev. Fluid Mech. 45, 269 (2013).

${ }^{70}$ T.D. Blake and J.M. Haynes, J. Colloid Interface Sci. 30, 421 (1969).

${ }^{71}$ R.G. Cox, J. Fluid Mech. 168, 169 (1986).

${ }^{72}$ H.J. van Ouwerkerk, Int. J. Heat Mass Transf. 14, 1415 (1971).

${ }^{73}$ H.J. Van Ouwerkerk, Chem. Eng. Sci. 27, 1957 (1972). 